\title{
Design of Magneto-Inductive Magnetic Resonance Imaging Catheters
}

\author{
Khoonsake Segkhoonthod, Richard R. A. Syms, Senior Member, IEEE, and Ian R. Young
}

\begin{abstract}
A catheter-based RF receiver for internal magnetic resonance imaging is described. The device consists of a doublesided thin-film circuit, mounted on a hollow catheter. The system was originally designed for biliary ductal imaging, but is also potentially useful for vascular imaging. Signals are detected using a resonant $\mathrm{L}-\mathrm{C}$ circuit at the catheter tip, transmitted along the catheter using an array of coupled $L-C$ resonators, and coupled into a conventional RF system using a demountable inductive transducer. Protection against external $B_{1}$ and $E$ fields is obtained by using figure-of-eight-shaped elements with an electrical length shorter than that of an immersed half-wave dipole. Electromagnetic modeling software (AWR Microwave Office) is used to analyze a system designed for ${ }^{1} \mathrm{H}$ imaging at 1.5 $T$, determine the effect of the tissue surround, demonstrate signal detection and transmission and verify intrinsic safety.
\end{abstract}

Index Terms - Microcoil, Magnetic resonance imaging, Magneto-inductive waveguide

\section{INTRODUCTION}

$\mathrm{B}$ ecause of their close proximity to the target tissue and restricted field of view for body noise, catheter based RF receivers can offer a significant local signal-to-noise ratio advantage over surface array coils in magnetic resonance imaging (MRI). A wide variety of catheter coils have therefore been developed for arterial imaging [1-6], or for tracking of catheter devices [7-9]. Despite this advantage, any devices containing conductors (including detection coils and output cables) that are immersed in tissue can induce potentially dangerous RF heating during the excitation phase of MRI.

Firstly, resonant loops may couple directly to the $\mathrm{B}_{1}$ field of the scanner's RF transmitter. The effect is minimized by the switched insertion of high impedance into the loop during excitation $[10,11]$. Secondly, surface waves [12] may be excited on extended lengths of conductor by electric fields, particularly near tuning capacitors in the transmitter coil. If these waves are resonant, the result may be rapid heating [13-

Manuscript received October 08, 2013.

Khoonsake Segkoonthod is with the EEE Dept., Imperial College London, Exhibition Road, London SW7 2AZ, UK.

Richard R. A. Syms is with the EEE Dept., Imperial College London, Exhibition Road, London SW7 2AZ, UK (phone: +44-207-594-6203; fax: +44-207-594-6308; e-mail: r.syms@,imperial.ac.uk).

Ian R. Young is with the EEE Dept., Imperial College London, Exhibition Road, London SW7 2AZ, UK.

Copyright (c) 2013 IEEE. Personal use of this material is permitted. However, permission to use this material for any other purposes must be obtained from the IEEE by sending an email to pubs-permissions@ieee.org.
16]. Unfortunately, the conductors are often closely surrounded by tissue, whose dielectric constant is high at standard clinical frequencies $\left(63.85 \mathrm{MHz}\right.$ for ${ }^{1} \mathrm{H} \mathrm{MRI}$ at $\left.1.5 \mathrm{~T}\right)$ [17], so the resonant length is short. Development of decoupling methods to control spurious resonances is more difficult for long conductors. Current approaches include insertion of RF traps [18, 19], transformer segmentation [20, 21] and reversal of conductor directions [22].

We have been studying catheter receiver designs for local MRI of the biliary ductal system. In this case, access may be provided by passing a catheter into the duct through the biopsy channel of a side-opening duodenoscope [23]. A long, smooth, flexible catheter is required, which must also contain a guide wire lumen to assist with cannulation. We have developed flexible thin-film circuits, which can be attached to the outside of a hollow catheter using heat-shrink tubing. Early versions used a printed coil and a coaxial output, while intermediate versions combined a printed coil and a printed cable. The most recent used a printed, transformer-segmented line known as a magneto-inductive (MI) waveguide. The catheters are small and flexible enough for endoscopic delivery, provide an image along their entire length, and are decoupled from both the $B_{1}$ and $E$ fields [24]. The approach is general enough to allow the addition of RF detection to many types of catheter.

Development has involved lumped element modeling and iterative experiment. However, the structures are distributed systems immersed in a surrounding medium and subjected to external fields, which cannot easily be described using equivalent circuits. The aim of this paper is therefore to provide a full electromagnetic simulation of signal detection and transmission, especially emphasizing decoupling and safety. MI waveguides and imaging devices are introduced in Section II. Commercial modeling software (AWR Microwave Office) is used to determine the effect of surrounding media in Section III, simulate signal detection and transmission in Section IV, and verify decoupling from external fields in Section V. Conclusions are presented in Section VI.

\section{MAGNeto-Inductive IMAGing CATHETERS}

In this section we briefly review the properties of magnetoinductive waveguides and MRI detectors, using low-frequency lumped element circuit theory.

\section{A. Magneto-inductive waveguides}

MI waveguides are linear arrays of magnetically coupled low-frequency $L-C$ resonators with internal resistances $R$ as shown in Fig. 1a [25]. 
a)

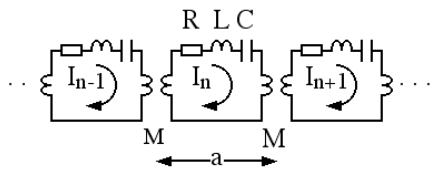

b)

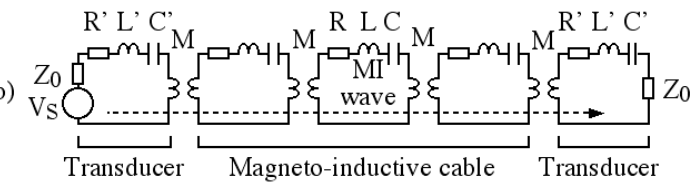

Fig. 1. Lumped element model of a) infinite magneto-inductive waveguide, and b) finite link between systems with real impedance $Z_{0}$.

Assuming nearest neighbour interaction, the resonators are coupled together by mutual inductance $M$. For an infinite guide, the currents $I_{\mathrm{n}-1}, I_{\mathrm{n}}$ and $I_{\mathrm{n}+1}$ at angular frequency $\omega$ are related using the recurrence relation:

$$
(R+\mathrm{j} \omega L+1 / \mathrm{j} \omega C) I_{\mathrm{n}}+\mathrm{j} \omega M\left(I_{\mathrm{n}-1}+I_{\mathrm{n}+1}\right)=0
$$

Assumption of wave solutions $I_{\mathrm{n}}=I_{0} \exp (-\mathrm{j} n k a)$, where $I_{0}$ is the wave amplitude, $k$ the propagation constant and $a$ the period, leads to the dispersion relation:

$$
1-\omega_{0}{ }^{2} / \omega^{2}-\mathrm{j} / Q+\kappa \cos (k a)=0
$$

Here, $\omega_{0}=1 / \sqrt{ }(L C)$ is the angular resonant frequency and $Q$ $=Q_{0} \omega / \omega_{0}$, where $Q_{0}=\omega_{0} L / R$ is the quality factor. Generally the propagation constant is complex. Writing $k=k^{\prime}-\mathrm{j} k^{\prime \prime}$ and assuming that $k^{\prime \prime}$ is small, one obtains:

$$
\begin{gathered}
\left(1-\omega_{0}{ }^{2} / \omega^{2}\right)+\kappa \cos \left(k^{\prime} a\right)=0 \\
k^{\prime \prime} a=1 /\left\{\kappa Q \sin \left(k^{\prime} a\right)\right\}
\end{gathered}
$$

The upper equation is the dispersion relation for loss-less MI waves. For positive $\kappa$, propagation is allowed only over the frequency band $1 / \sqrt{ }(1+\kappa) \leq \omega / \omega_{0} \leq 1 / \sqrt{ }(1-\kappa)$. $k^{\prime} a$ tends to zero and $\pi$ at the lower and upper band edges. The effect of finite Q-factor is to introduce loss and allow out-of-band propagation. The lower equation is the approximate loss variation. Loss is minimized at resonance (when $k^{\prime} a \approx \pi / 2$ ) and inversely proportional to both $\kappa$ and $\mathrm{Q}_{0}$. The characteristic impedance is $\mathrm{j} \omega M \sin (k a)$, which for low loss reduces approximately to the real value $Z_{0 \mathrm{M}}=\omega_{0} M$ at resonance.

MI waveguides have been demonstrated experimentally, but it has been difficult to achieve good performance. Low propagation loss requires resonators with high Q-factors that are strongly coupled to next neighbours. Small variations in the mutual inductance cause reflection. However, stability is achieved using a thin-film cable that can be flexed without changing $M$ [26]. Practical operation also requires transducers for coupling to RF systems with real impedance $Z_{0}$. These can be realized using additional resonant loops with parameters $R^{\prime}$, $L^{\prime}$ and $C^{\prime}$ as shown in Fig. 1b. Provided $Z_{0 \mathrm{M}}=Z_{0}$, efficient power transfer can be achieved over most of the MI band if $L^{\prime}$ $=L / 2$ and $C^{\prime}=2 C$. Matching may still be achieved if $Z_{0 \mathrm{M}} \neq Z_{0}$, but only over a narrow range. Finally, stray capacitance between overlaid elements leads to electric coupling, introducing unwanted high frequency propagation [27].

\section{B. Magneto-inductive detectors}

Fig. 2 shows a magneto-inductive receiver, which consists of a MI waveguide that is impedance matched to the scanner at one end and self-terminating at the other. The parameters $L$ and $C$ are chosen so that $\omega_{0}$ is the Larmor frequency. At this frequency, $Z_{0 \mathrm{M}}$ will not always correspond to the scanner's impedance $Z_{0}$. However, matching can be achieved using a transducer loop, which in general contains inductance $L^{\prime}$ and capacitance $C^{\prime}$ and is also resonant at $\omega_{0}$. The transducer is coupled to the waveguide via mutual inductance $M^{\prime}$, and matching is achieved when $\omega_{0}{ }^{2} M^{2}=Z_{0} Z_{0 \mathrm{M}}$. Self-termination is achieved by adjusting the mutual inductance $M^{\prime \prime}$ between the final element and the detector so that $\omega_{0}^{2} M^{\prime 2}=R Z_{0 \mathrm{M}}$. In this case, a signal $V_{\mathrm{S}}$ generated by the scanner will excite a MI wave that travels down to the detector without reflection as shown in Fig. 2a. Conversely, a signal in the detector loop will excite a wave that travels back to the scanner.

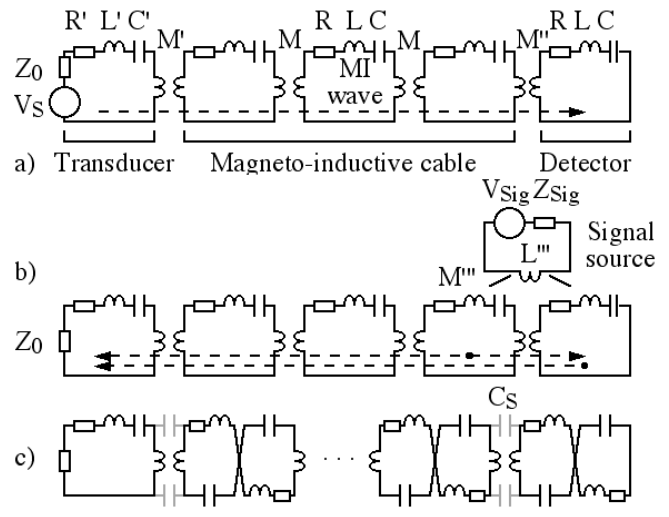

Fig. 2. Lumped element models to demonstrate a) impedance matching, b) imaging and c) figure-of-eight elements with spurious capacitance.

MR signals will of course be magnetically induced. This process may be represented as shown in Figure $2 b$. Here a small loop with impedance $Z_{\text {sig }}$ containing a voltage $V_{\text {sig }}$ models a group of precessing dipoles. The signal may couple to any of the receiver elements. Because of this, the receiver can act as a detector along its entire length. Depending on the arrangement, the signal may couple to more than one element. In the example here, the signal couples to the detector and the final waveguide element via a mutual inductance $M^{\prime \prime}$. Several waves are therefore excited. The wave excited in the detector travels to the scanner input as before. However the voltage induced in the waveguide element generates two counterpropagating waves. One travels to the scanner input, while the other is absorbed in the detector. Thus, in general, the detection process is relatively complex.

\section{Intrinsic safety}

Further modifications are required to introduce intrinsic safety. Firstly, the resonators must be prevented from coupling directly to $B_{1}$ fields. The use of segmentation and the need for catheter integration make it difficult to apply conventional methods based on impedance switching. However, because $B_{1}$ fields are generally uniform, some inherent decoupling can be provided by twisting each element into a figure of-eight shape as shown in Fig. 2c, so that any voltages induced in the two halves cancel. Secondly, the line must be prevented from coupling to external $E$-fields. The frequency of the $v^{\text {th }}$ order 
resonance of a length $d$ of conductor should lie at:

$$
f_{\mathrm{v}}=v c /\left(2 d \sqrt{ } \varepsilon_{\mathrm{r}}\right)
$$

Here $v$ is an integer, $c$ is the velocity of light, and $\varepsilon_{\mathrm{r}}$ is the effective relative permittivity of the surround. Assuming that $\varepsilon_{\mathrm{r}}=77$ (a representative value for tissue), $f_{1}$ will lie at 63.85 $\mathrm{MHz}$ when $d \approx 27 \mathrm{~cm}$ [18]. The line should therefore be subdivided into lengths shorter than this value. However, even then, there may be a spurious capacitance $C_{\mathrm{S}}$ between sections as shown in Fig. 2c, which reduces the effect of segmentation. $C_{\mathrm{S}}$ is reduced by avoiding exact overlay of the windings. However, the price is a reduction in magnetic coupling, which from (3) translates into an increase in loss.

\section{Thin-film realization}

The circuit in Fig. 2c can be realized in thin-film form using copper-clad polyimide patterned using double-sided exposure and etching. Fig. 3a shows a suitable layout. Each inductor is formed in one layer of metal using a track of width $w_{\mathrm{L}}$, as a figure-of-eight shape of length $L_{\mathrm{L}}$ and width $W_{\mathrm{L}}$. However, the capacitors are divided into two series-connected components of length $L_{\mathrm{C}}$ and width $W_{\mathrm{C}}$ that use the substrate as an interlayer. The inductors from adjacent elements occupy different sides of the substrate, so that they may be overlaid with their nearest neighbours, but are offset to minimize $C_{\mathrm{S}}$.

a)

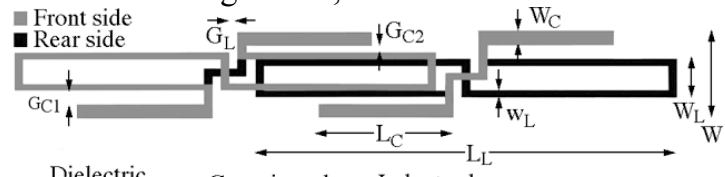

b)
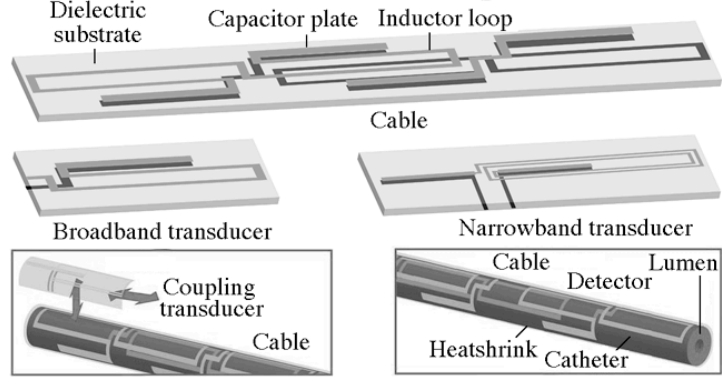

Fig.3 Thin-film detector: a) and b) circuit, and c) integration on catheter.

Fig. $3 b$ shows three-dimensional representations of the resulting cable, together with a broadband transducer (a halved cable element) and a narrow-band transducer (a two-turn spiral inductor made resonant with a pair of integrated capacitors). The former may be used when $Z_{0 \mathrm{M}} \approx Z_{0}$, and the latter when $Z_{0 \mathrm{M}} \neq Z_{0}$ and larger mutual inductance is required. A complete detector consists of $N$ elements of MI cable, with one similar element (whose position is chosen to provide selftermination) forming the detector. The circuit is mounted on a hollow catheter as shown in Fig. 3c. The transducer is demountable, allowing $M^{\prime}$ to be varied mechanically.

To pass a biopsy channel, the catheter diameter must be small, typically $\approx 3 \mathrm{~mm}$. To allow sufficient flexibility, the conductor and dielectric layers must be thin. Suitable performance has been obtained using $35 \mu \mathrm{m} \mathrm{Cu}$ on $25 \mu \mathrm{m}$ polyimide [24]. The overall width $W$ of the circuit must be less than the circumference of the scaffold, with the inductor width $W_{\mathrm{L}}$ chosen to place the long conductors on either side of a diameter. The capacitor width $W_{\mathrm{C}}$ must then be adjusted to fit the capacitors into the remaining space. Gaps $G_{\mathrm{C} 1}$ and $G_{\mathrm{C} 2}$ are needed to avoid further parasitic capacitance. The lengths $L_{\mathrm{L}}$ and $L_{\mathrm{C}}$ of the inductors and capacitors must yield the correct resonant frequency, and the conductor width $w_{\mathrm{L}}$ must ensure a high Q-factor. The length $L_{\mathrm{L}}$ of each section must be less than the critical resonant length. The number of elements should yield a total length $(N+2) L_{\mathrm{L}} / 2$ sufficient to pass through a duodenoscope $(\mathrm{ca} 2 \mathrm{~m})$. Suitable dimensions for catheters operating at $63.8 \mathrm{MHz}$ are summarized in Table I.

\begin{tabular}{|c|c|c|}
\hline Parameter & Actual value & Model value \\
\hline$W(\mathrm{~mm})$ & 7.75 & 6.75 \\
\hline$W_{\mathrm{L}}(\mathrm{mm})$ & 5.00 & 4.00 \\
\hline$W_{\mathrm{C}}(\mathrm{mm})$ & 0.75 & 0.75 \\
\hline$w_{\mathrm{C}}(\mathrm{mm})$ & 0.50 & 0.50 \\
\hline$L_{\mathrm{L}}(\mathrm{mm})$ & 196.50 & 196.50 \\
\hline$L_{\mathrm{C}}(\mathrm{mm})$ & 49.25 & 49.25 \\
\hline$G_{\mathrm{C} 1}(\mathrm{~mm})$ & 0.875 & 0.875 \\
\hline$G_{\mathrm{C} 2}(\mathrm{~mm})$ & 0.375 & 0.375 \\
\hline$G_{\mathrm{L}}(\mathrm{mm})$ & 0.50 & 0.50 \\
\hline
\end{tabular}

Table I. Dimensional parameters in Fig. 3 used in the simulations.

\section{Modelling Overview, And Cladding Materials}

To reduce run-times, was assumed that the circuit is entirely flat (rather mounted on a catheter, or bent as in cannulation), thus avoiding the need for full 3D simulation. Modeling was also carried out using a reduced number (7) of elements in the MI cable. These assumptions allow rapid exploration of layouts, while yielding realistic results. Simulation was carried out with commercial electromagnetic analysis software (Microwave Office, AWR Corp., El Segundo, CA, USA), using the Axiem ${ }^{\circledR}$ 3D planar method-of-moments solver.

\section{A. AWR Microwave Office}

Axiem assumes a multilayer structure for computation. Initially, four-layer structures containing a single metal layer capable of defining simple wires were modeled, as shown in Fig. 4a. Here, layers 1 and 5 are assumed to be uniform polyolefin, with thickness $t_{1}$ and $t_{5}$ and complex relative permittivity $\varepsilon_{\mathrm{rC}}=\varepsilon_{\mathrm{rC}}{ }^{\prime}-\mathrm{j} \varepsilon_{\mathrm{rC}}{ }^{\prime \prime}$. The former mimics the catheter scaffold and the latter the heat-shrink. Layer 2 is assumed to be metal, with thickness $t_{2}$ and conductivity $\sigma$, and patterned to define an in-plane conductor layout. To allow modeling of tissue immersion, layer 6 was assumed to be a layer of tissue, with thickness $t_{6}$ and complex relative permittivity $\varepsilon_{\mathrm{rT}}=\varepsilon_{\mathrm{rT}}{ }^{\prime}$ $\mathrm{j} \varepsilon_{\mathrm{rT}}$ ".

Six-layer structures containing a second metal layer to allow definition of a full circuit were then investigated, as shown in Fig. 4b. Here layer 3 is assumed to be a uniform polyimide interlayer, with thickness $t_{3}$ and relative permittivity $\varepsilon_{\mathrm{rI}}=\varepsilon_{\mathrm{rI}}{ }^{\prime}-\mathrm{j} \varepsilon_{\mathrm{rI}}{ }^{\prime \prime}$. Layer 4 is assumed to be metal, with thickness $t_{4}$ and conductivity $\sigma$, and may be patterned to define a separate conductor layout. Finally, once the effect of immersion had been established, simpler five-layer structures omitting the tissue were investigated as shown in Fig. 4c. 

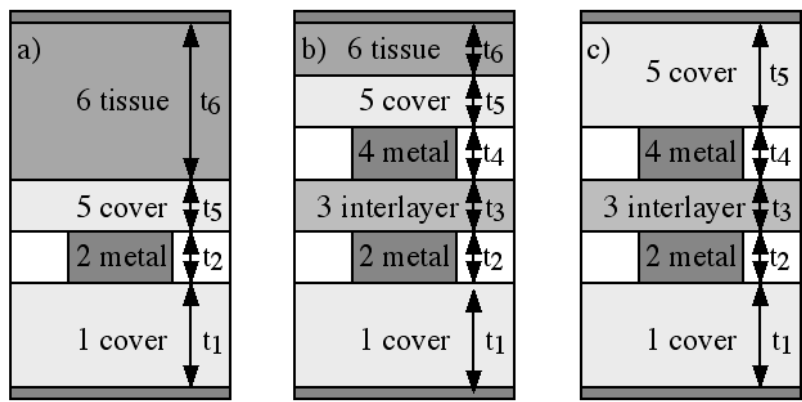

Fig. 4 a) plastic-embedded single-layer metal, with tissue layer; b) and c) plastic-embedded double-layer metal, with and without tissue.

Different devices were simulated, by specifying an appropriate metal layout and ports connecting to sources and loads, each of $50 \Omega$ impedance. The assumed values of the material parameters are shown in Table II. Typically, these are as found in the literature, but the conductivity was reduced from the value obtained in bulk copper $\left(\sigma=5.96 \times 10^{7} \mathrm{~S} / \mathrm{m}\right)$ to mimic the relatively poor performance of thin film $\mathrm{Cu}$.

\begin{tabular}{|c|c|c|}
\hline Material & Conductivity $\sigma(\mathrm{S} / \mathrm{m})$ & \\
\hline Copper & $2.96 \times 10^{7}$ & \\
\hline Material & Real permittivity $\varepsilon_{\mathrm{r}}{ }^{\prime}$ & $\operatorname{Tan}(\delta) \varepsilon_{\mathrm{r}}{ }^{\prime \prime} / \varepsilon_{\mathrm{r}}{ }^{\prime}$ \\
\hline Polyolefin & 2.7 & 0.06 \\
\hline Polyimide & 3.5 & 0.06 \\
\hline Tissue & 77 & 0 \\
\hline
\end{tabular}

Table II. Material parameters used in the simulations.

\section{B. Immersed wires}

To begin with, the effect of tissue immersion was simulated using the structure of Fig. $4 \mathrm{a}$, with the aim of clarifying the effects of a plastic cover on surface waves. The metal pattern was first chosen to define a long wire of width $0.25 \mathrm{~mm}$, with a pair of weakly coupled short dipoles $1.65 \mathrm{~mm}$ away from the wire at either end for electrical excitation and detection as shown in Fig. 5a. Calculation of the frequency variation of transmission $\left(S_{21}\right)$ then yielded a response containing resonant peaks, each at a frequency $f_{v}$ corresponding to the $v^{\text {th }}$ order resonance of the wire. Knowledge of (say) $f_{1}$ then allowed the effective value of $\varepsilon_{\mathrm{r}}$ (a weighted average of the permittivity of all the surrounding layers) to be estimated using Equation 4.

The thickness $t_{1}$ and $t_{6}$ of the polyolefin substrate and tissue were both taken as $30 \mathrm{~mm}$, the thickness $t_{2}$ of the copper was $35 \mu \mathrm{m}$, and the thickness $t_{5}$ of the polyolefin cover was varied. Fig. $5 \mathrm{~b}$ shows the variation of $\varepsilon_{\mathrm{r}}$ with $t_{5}$. When $t_{5}=0$, a value of $\varepsilon_{\mathrm{r}}=39.1$ was obtained. This value is almost exactly equal to $\left(\varepsilon_{\mathrm{r} 1}+\varepsilon_{\mathrm{r} 6}\right) / 2$, implying that the relative permittivity seen by the wire is the average of the values of the polyolefin support and the tissue surround. However, as $t_{5}$ increases, $\varepsilon_{\mathrm{r}}$ falls towards 2.7, the value for complete immersion in polyolefin. The initial rapid reduction in $\varepsilon_{\mathrm{r}}$ implies that even a thin cover is sufficient to reduce the wavelength-shortening effect of surrounding tissue significantly. Consequently, designs based on full immersion in tissue and implemented with (say) a 250 $\mu \mathrm{m}$ thick heat-shrink tube will be extremely conservative. a)
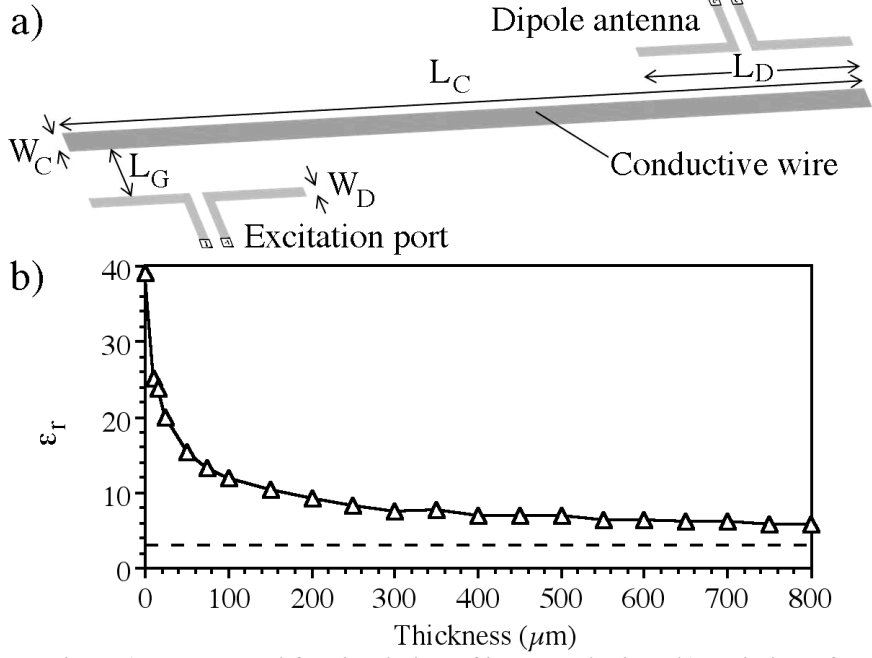

Fig. 5 a) Layout used for simulation of immersed wires; b) variation of effective relative dielectric constant with cover layer thickness.

\section{Parasitic capacitance}

The origin of the parasitic capacitance $C_{\mathrm{S}}$, and the effect of tissue immersion on $C_{\mathrm{S}}$, was then simulated using the doublelayer metal structure of Fig. $4 \mathrm{~b}$. The thickness of the additional layers were $t_{3}=25 \mu \mathrm{m}$ (polyimide interlayer) and $t_{4}$ $=35 \mu \mathrm{m}$ (second $\mathrm{Cu}$ layer). Fig. 6a shows a typical arrangement. Here, conductors on the two layers have been offset to avoid parallel-plate effects, but electric coupling is still obtained through fringing fields that penetrate multiple layers of dielectric. As a result, $C_{\mathrm{S}}$ may be large if significant field exists in the tissue, which has a high dielectric constant. Problems of this type are important in multilayer VLSI circuits. Unfortunately, analytic evaluation of the capacitance is extremely difficult. Solutions for infinite, asymmetrically placed strips in uniform dielectrics have been obtained using conformal mapping [28], and similar approaches have been adopted for more complex geometries [29]. However, the problem must generally be tackled numerically.

a)

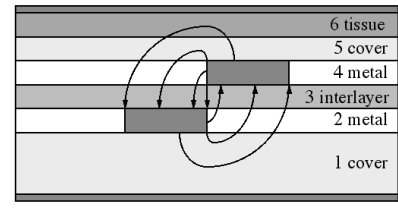

\section{b)}
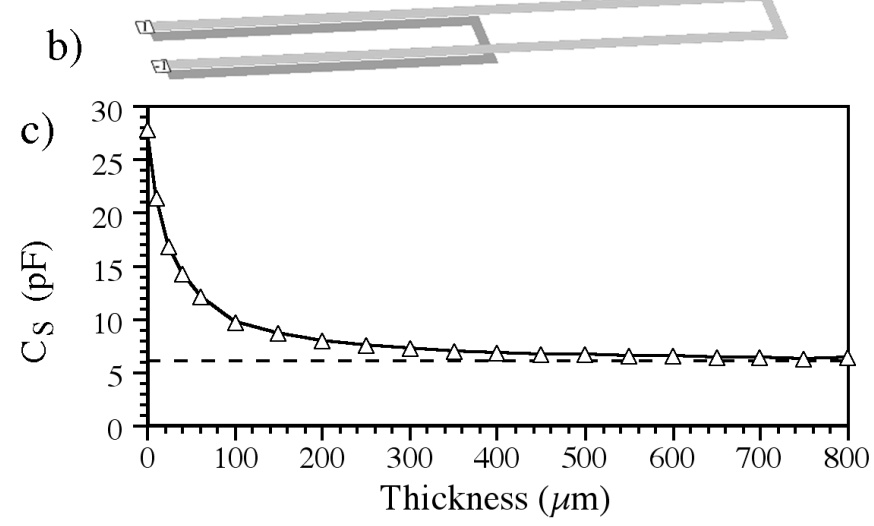

Fig. 6 a) Origin of electric coupling; b) layout for simulation of parasitic capacitance; c) variation of $C_{\mathrm{S}}$ with cover layer thickness.

Here, we have determined the parasitic capacitance using a 
resonant circuit based on two U-shaped metallic loops provided on the two available metal layers as shown in Fig. $6 \mathrm{~b}$. The right-hand part of the circuit defines an inductor, whose value may be determined using a known capacitance, while the left-hand part defines two series-connected parasitic capacitors. The circuit response is probed using ports attached to the capacitors, and their value is extracted from the resonant frequency. Fig. $6 \mathrm{c}$ shows the variation of $C_{\mathrm{S}}$ with the cover layer thickness $t_{5}$. Once again, $C_{\mathrm{S}}$ falls rapidly from an initial value $C_{\mathrm{S} 0}$ when $t_{5}=0$, stabilizing when $t_{5}$ rises to a few hundred microns at the value obtained for an infinitely thick cladding. We may therefore deduce that a plastic heat-shrink also provides an effective shield against the effect of surrounding tissue on fringing fields. Further simulations were therefore carried out using the layer structure of Fig. 4c, which omits the tissue layer entirely, and instead assumes a $30 \mathrm{~mm}$ thick layer of polyolefin above the circuit.

\section{SignAl TRANSMISSION AND DETECTION}

We now consider specific circuits of the type shown in Fig. 3. Layout dimensions largely follow those of the experimental devices given in Table I, but with some small differences. Particularly, 2D simulation of 3D inductors meant that exactly corresponding values of self- and mutual inductance could not be obtained simply by adopting the same inductor width, and slightly modified values were therefore used. These were obtained by trial-and-error, and are as detailed in Table I.

\section{A. Single and coupled resonant elements}

The analysis of Sec. II implies that the properties of MI waveguides largely follow from the resistance $R$, inductance $L$ and capacitance $C$ of a single element and the mutual inductance $M$. Their effect on dispersion and loss can be summarized in terms of the angular resonant frequency $\omega_{0}=$ $1 / \sqrt{ }(L C)$, the coupling coefficient $\kappa=2 M / L$ and the Q-factor $Q_{0}=\omega_{0} L / R$. Approximate values of $\omega_{0}, \kappa$ and $Q_{0}$ were known from comparisons between experimental data and MATLAB models of the circuits in Figs. 1 and 2. Equivalent values were established in AWR using techniques that mimic experimental methods.

Two simple AWR layouts were first defined as shown in Fig. 7a. The upper one is a single resonant element, interrogated inductively using two weak taps. The lower one is a pair of coupled resonant elements, again interrogated inductively. Simulation of the frequency variation of transmission through the upper layout allowed the resonant frequency $f_{0}=\omega_{0} / 2 \pi$ and the quality factor $Q_{0}$ of a single element to be found from the position and $3 \mathrm{~dB}$ bandwidth of the resonance. Similarly, simulation of the lower layout allowed the two resonant frequencies $f_{1}=1 /\{2 \pi \sqrt{ }[(L+M) C]\}$ and $f_{2}=1 /\{2 \pi V[(L-M) C]\}$ inherent in a coupled system to be identified. These values allowed the coupling coefficient to be estimated as $\kappa=2\left\{f_{2}^{2} / f_{1}^{2}-1\right\} /\left\{f_{2}^{2} / f_{1}^{2}+1\right\}$.

In each case, the inductor width $W_{\mathrm{L}}$ and the capacitor length $L_{\mathrm{C}}$ were initially assumed to have the experimental values, and the conductivity to have the value for bulk copper. The inductor width $W_{\mathrm{L}}$ was first adjusted to obtain the correct value of $\kappa$. The capacitor length $L_{\mathrm{C}}$ was then adjusted to obtain the correct value of $f_{0}$. Finally, the conductivity was adjusted to obtain the correct Q-factor.
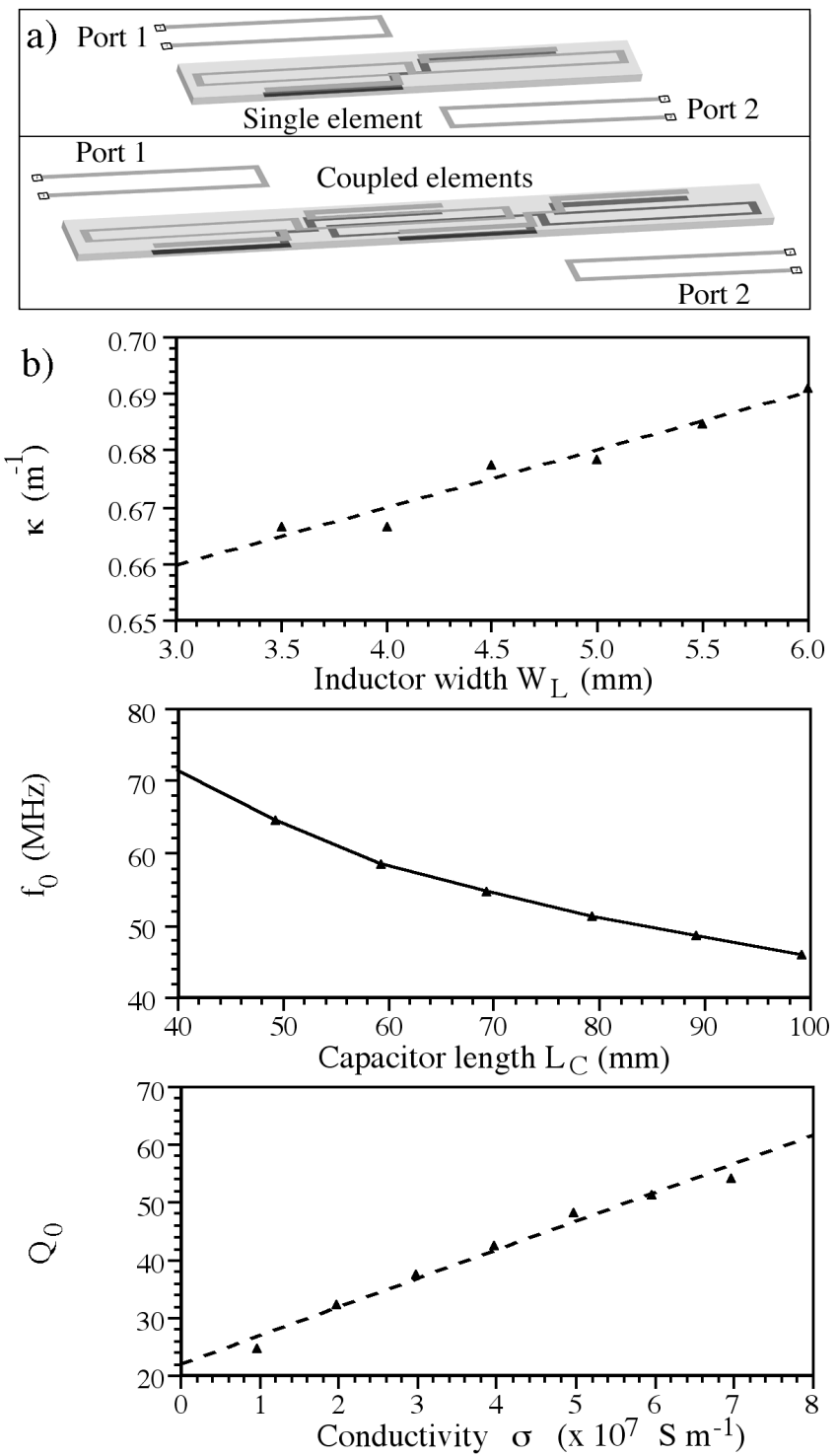

Fig. 7 Parameter extraction: a) AWR models; b) variation of $\kappa$ with $\mathrm{W}_{\mathrm{L}}, \mathrm{f}_{0}$ with $\mathrm{L}_{\mathrm{C}}$ and $\mathrm{Q}_{0}$ with $\sigma$.

Simulations were carried out using air and polyolefin as surrounds. In contrast to the previous section on electric effects, little difference was obtained in the parameter values of the components (whose behaviour is dominated by magnetic effects). Fig. $7 \mathrm{~b}$ shows the variations of $\kappa, f_{0}$ and $Q_{0}$ with $W_{\mathrm{L}}, L_{\mathrm{C}}$ and $\sigma$, and Table III details the final values.

\begin{tabular}{|l|l|l|l|}
\hline Parameter & $\kappa$ & $\mathrm{f}_{0}$ & $\mathrm{Q}_{0}$ \\
\hline Value & 0.684 & 63.90 & 40.96 \\
\hline \multicolumn{3}{|r}{ Table III. Performance parameters of MI waveguides. }
\end{tabular}

\section{B. Complete magneto-inductive systems}

Complete links could then be simulated. For example, Fig. 8a shows an AWR model equivalent to Fig. 1b. Here a length of cable is connected at either end to input and output ports using halved cable elements acting as a broadband transducers. 
Fig. 8b compares the frequency variation of transmission and reflection $\left(S_{21}\right.$ and $\left.S_{11}\right)$ predicted using the AWR model and a MATLAB solution of the governing equations for Fig. $1 \mathrm{~b}$. The transmission is low, except in the MI band from 50 $\mathrm{MHz}$ to $120 \mathrm{MHz}$ where it reaches around $-5 \mathrm{~dB}$. The agreement between the two models is generally good, except at high frequency, where it rises in the AWR model. When the same data is plotted over an extended frequency range, it is clear that this effect can be attributed to an additional propagation band in the AWR model, which is not predicted using the simple MI waveguide model. This band is due to parasitic capacitance between closely spaced tracks where the inductors are overlaid to achieve strong magnetic coupling, and has the additional effect of reducing the width of the MI band somewhat [27]. The predicted pass-band is reduced from $50-105 \mathrm{MHz}$ to $50-75 \mathrm{MHz}$, and the predicted loss at 63.85 $\mathrm{MHz}$ is increased by $3 \mathrm{~dB}$. Similarly, the reflection is generally high except in the MI band. For both models, $S_{11} \approx-$ $20 \mathrm{~dB}$ over a reasonable frequency range, indicating that broadband impedance matching has been achieved.
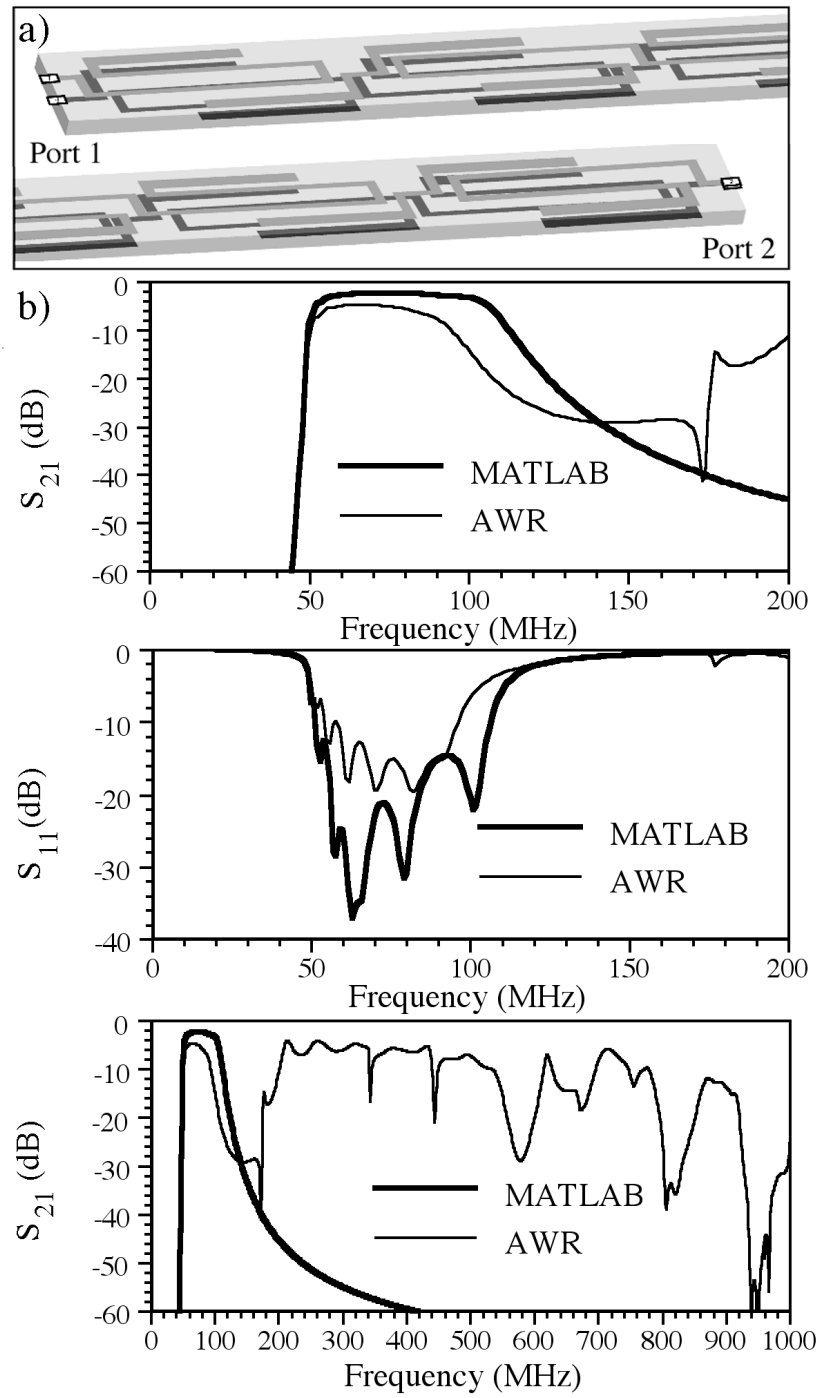

Fig. 8 Cable link simulation: a) AWR model; b) frequency variations of $\mathrm{S}_{21}$ and $\mathrm{S}_{11}$ predicted by AWR and MATLAB, over different frequency ranges.

Complete magneto-inductive detectors could then be simulated. For example, Fig. 9a shows an AWR model equivalent to Fig. 2b. Here a length of cable is connected at one end to an input port using a spiral resonant inductor, which acts as a narrow-band transducer, and at the other end using an element whose position is adjusted to form a matched termination. A weak inductive tap is also provided, to allow transmission to be simulated. In this case, additional dimensional adjustments were required to achieve the correct electrical parameters. The lengths of the capacitors in the transducer were first chosen to set the resonance correctly. The positions of the transducer and detector were then adjusted to achieve impedance matching at either end.

Fig. 9b shows the frequency variation of $S_{21}$ and $S_{11}$ predicted using the AWR model and a MATLAB solution of the governing equations for Figure $2 \mathrm{~b}$. In the former case, the data were offset by a fixed amount to compensate for the use of a weak tap for sampling. The agreement between the two models is again good. There is again transmission over the MI band, but signal now peaks sharply at the resonant frequency indicating that the system is now functioning as a resonant detector with a $\mathrm{Q}$-factor of $\approx 40$. The predicted pass-band is again reduced as described with reference to Fig. 8. There is also a slight detuning of the resonant peak (by $3 \mathrm{MHz}$ ), which would in practice require mechanical tuning of the detector element. For both models, $S_{11} \approx-20 \mathrm{~dB}$ near the resonant frequency, indicating that narrow-band impedance matching has been achieved.
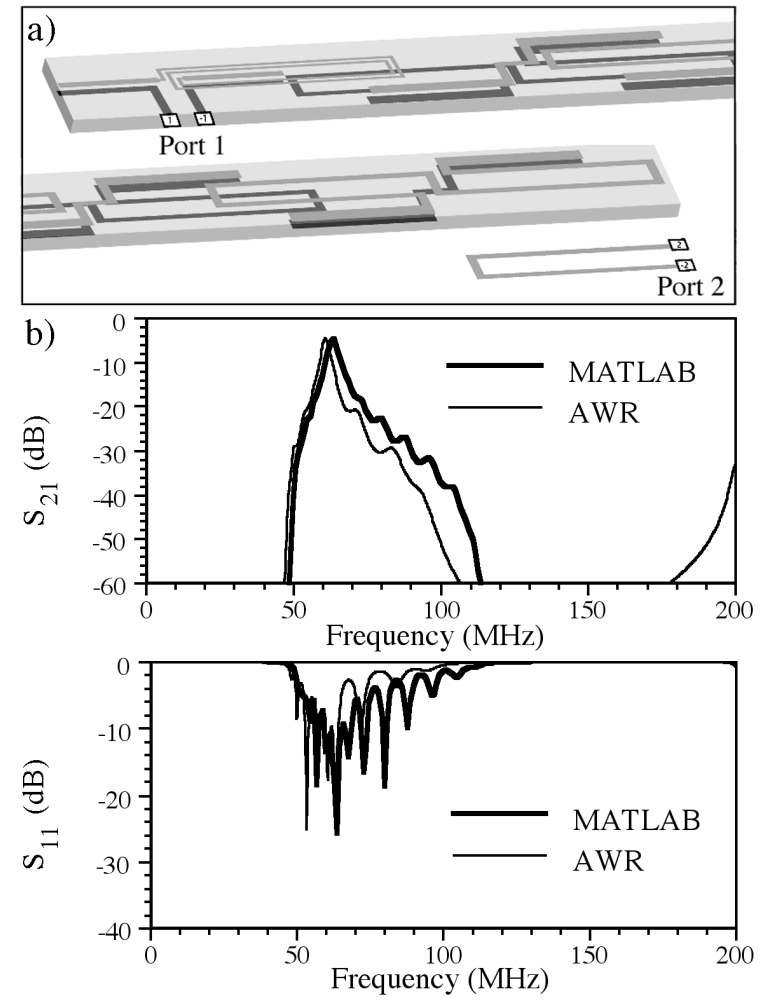

Fig. 9 Detector simulation: a) AWR model; b) frequency variations of $S_{11}$ and $\mathrm{S}_{21}$ as predicted by AWR and MATLAB.

\section{DECOUPLING FROM EXTERNAL FIELDS}

We now consider decoupling from external fields. Additional structures are required to generate suitable $B_{1}$ and $E$ fields and detect the resulting response. 


\section{A. Magnetic fields}

For magnetic fields, external excitation and detection can be carried out using inductive loops as shown in Fig. 10a. In the left-hand diagram, an offset loop on the left-hand side is used to excite a single resonant element of the magneto-inductive cable, and a similar loop on the right-hand side is used to detect any current flowing as a result of magnetic induction. In this case the excitation is asymmetric, and the frequency variation of the transmission $S_{21}$ between the loops displays a pronounced resonance at $63.85 \mathrm{MHz}$, as shown in Fig. 10b. In the right-hand diagram in Fig. 10a, the excitation loop is now placed centrally. In this case the excitation is symmetric, and the resonance is largely absent in the corresponding frequency variation in Fig. 10b due to cancellation of the induction. The figure-of-eight layout therefore provides protection against balanced external magnetic fields, but not unbalanced fields.

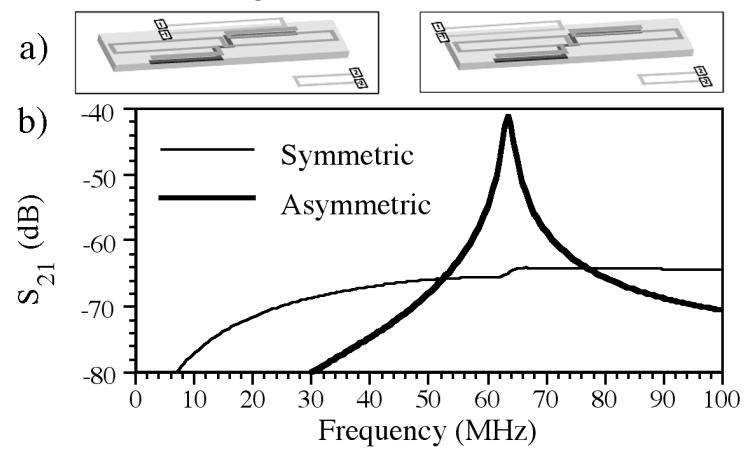

Fig. 10 Simulation of magnetic decoupling: a) AWR models, and b) frequency variations of $\mathrm{S}_{21}$ for asymmetric and symmetric excitation.

\section{B. Electric fields}

For electric fields, external excitation and detection can be carried out using antennas as shown in Fig. 11a. Excitation was carried out using a short (a tenth of a wavelength long) dipole at the left-hand end of the structure, a distance of 12.5 $\mathrm{mm}$ beneath. Detection of any excited currents was carried out using a similar dipole placed at the right-hand end, $12.5 \mathrm{~mm}$ above. Two structures were compared: an undivided wire of width $2.0 \mathrm{~mm}$ and thickness $0.35 \mu \mathrm{m}$ (upper diagram) and a magneto-inductive cable (lower diagram), in each case of length corresponding to 7 cable sections. Simulations were carried out with the structure under test covered with a dielectric of relative permittivity $\varepsilon_{\mathrm{r}}=77$, firstly with no cladding at all and secondly with a $30 \mathrm{~mm}$ polyolefin lower cladding and a $250 \mu \mathrm{m}$ thick polyolefin upper cladding. The upper diagram in Fig. 11b shows the frequency variation of transmission $S_{21}$ for wires. With no cladding, many resonances can be seen, extending as low as $20 \mathrm{MHz}$. The effect of the cladding is to decrease the effective permittivity seen by the wire, and significantly increase the frequency of the lowest order resonance.

The lower diagram in Fig. 11b shows the corresponding frequency variation for the MI cable. The effect of subdivision is, as expected, to raise the frequency of the lowest order resonance. However, the relatively large value of parasitic capacitance $C_{\mathrm{S}}$ obtained for the unclad system limits the effect of subdivision, so that the lowest order resonance actually lies below 63.85 MHz. The effect of the cladding is simultaneously to decrease the effective permittivity seen by conductors and to reduce $C_{\mathrm{S}}$. In this case, there are no significant resonances up to at least $150 \mathrm{MHz}$, and the attenuation in transmission is very substantial indeed. The segmented arrangement then provides extremely effective protection against external electric fields.
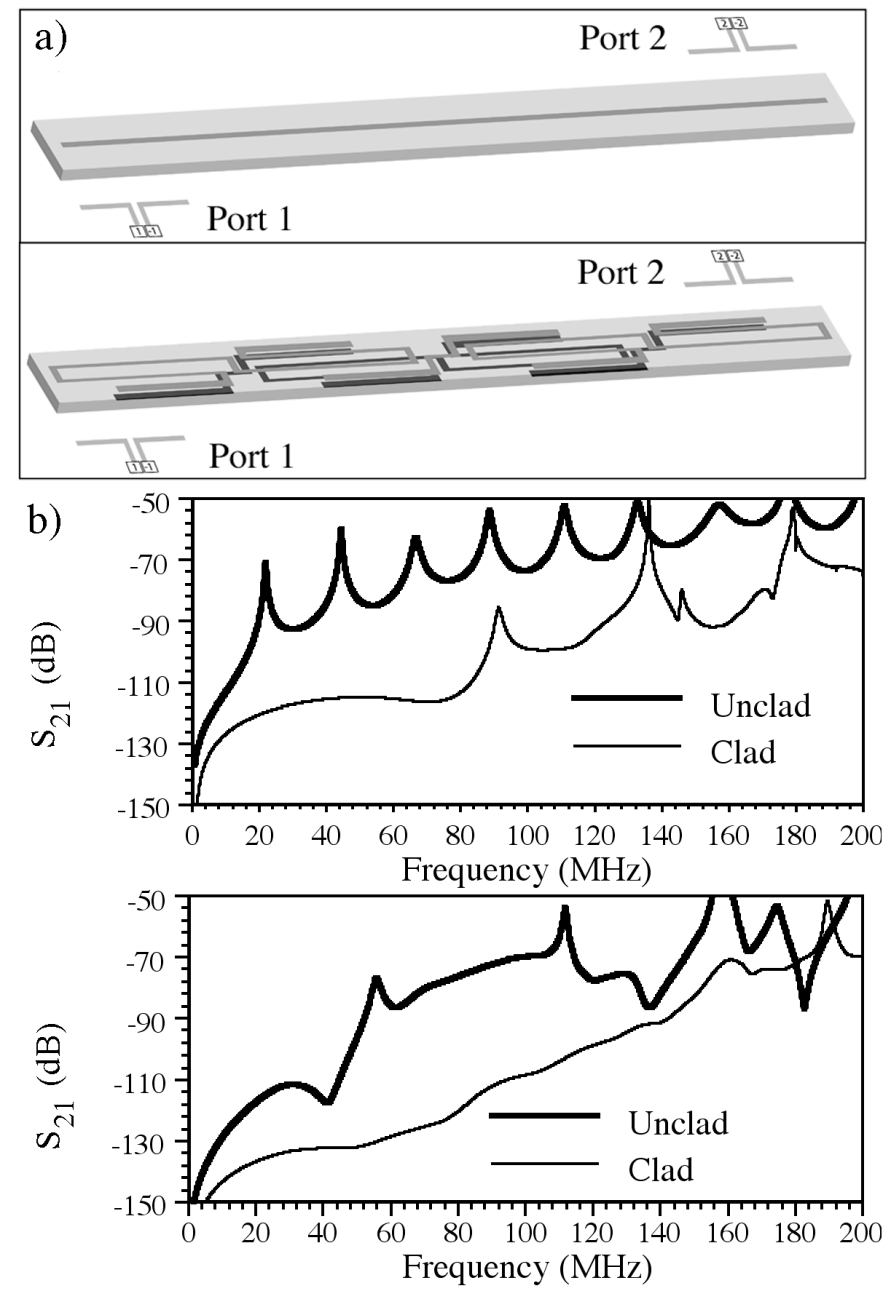

Fig. 11. Simulation of electric decoupling: a) AWR models and b) frequency variations of $\mathrm{S}_{21}$ for wire and cable, with and without cladding.

\section{CONCLUSIONS}

A detailed investigation of the thin-film circuit used in a catheter probe for high-resolution internal imaging has been carried out using commercial software (AWR Microwave Office). The circuit consists of a self-terminating magnetoinductive waveguide, formed from a set of magnetically coupled L-C resonators with inductors and capacitors that are entirely fabricated by double-sided patterning and etching of copper-clad Kapton. The resonant-elements are formed into figure-of-eight loops to provide inherent rejection of external $B_{1}$ fields, and the length of each element is chosen to be less than the resonant length of an immersed dipole at the operating frequency to avoid excitation of standing waves by external $E$ fields. The circuit is wrapped around a catheter and sealed in place with heat shrink tubing, and connection to the circuit is made using an inductive coupling transducer. 
Potential applications include biliary and arterial imaging.

The catheter architecture is an advance on previous internal probe designs, since it leaves the catheter lumen free for use with the guide-wire needed for reliable cannulation in biliary imaging. However, the position of the thin-film circuit leaves it separated from external tissue only by a thin insulation layer. It is therefore important to understand the precise effect of this layer on MRI safety. This investigation represents the first detailed simulation of such external effects.

The numerical simulations verify previous lumped element circuit analysis. Physical dimensions that give suitable component values have been established, subject to known layout constraints and simplification to a flat circuit. Matching between the MI waveguide and the scanner input has been achieved using a transformer, and matching between the final element of the waveguide and the detector by adjusting their separation. Operation of MI links and receivers has been simulated. The effect of parasitic capacitance between resonant elements has been quantified, and shown to allow additional high-frequency pass-bands for differential mode signals. The effect of an external medium has been clarified. The results show clearly that the heat-shrink tubing used to attach the circuit to the catheter scaffold serves the important function of reducing the effective permittivity of the surround and minimizing the value of parasitic capacitances. As a result, the circuit is indeed decoupled from external $B_{1}$ and $E$ fields and intrinsically safe.

High-resolution imaging with experimental catheter receivers has already been described [24]. Preliminary ${ }^{1} \mathrm{H}$ MR safety experiments have also been carried out, using a $1.5 \mathrm{~T}$ GE Signa Excite scanner. MI cables with 2, 3 and 4 sections were suspended in a viscous gel based on polyacrylic acid and saline designed to mimic human tissue as shown in Fig. 12a.

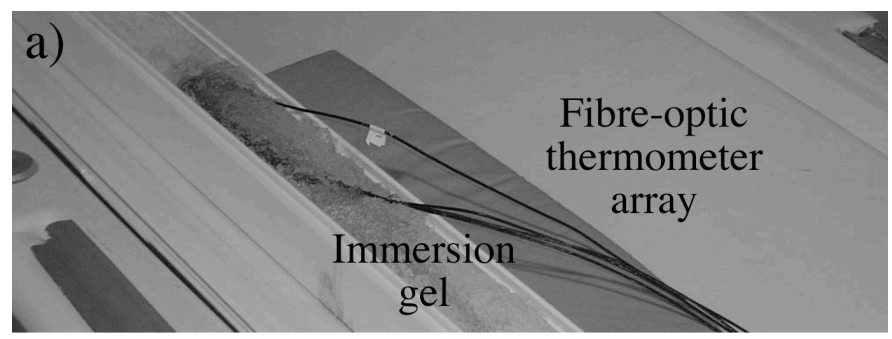

b)

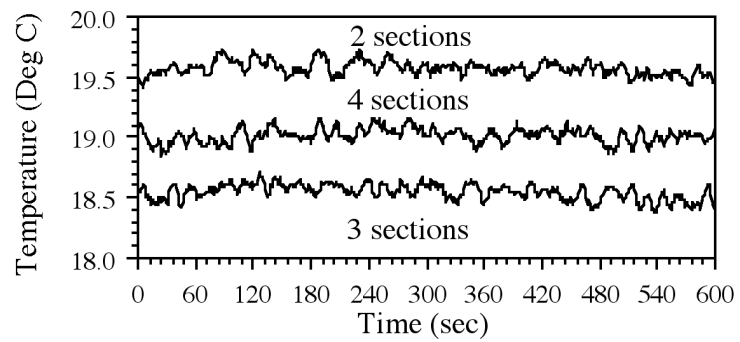

Fig. 12 a) Arrangement for experimental measurement of RF-induced heating effects; b) time evolution of temperature.

The temperature was monitored at the cable midpoints and ends with a multi-channel fibre optic temperature probe while scanning with a RF-intensive FIESTA sequence $\left(120^{\circ}\right.$ flip angle, echo time $\mathrm{TE}=2.3 \mathrm{~ms}$, repeat time $\mathrm{TR}=8 \mathrm{~ms}$ ) with the cables close to the system body coil. Fig. 12b shows the time evolution of the midpoint temperature. Here, differences between probes are attributable to calibration errors. The lack of any temperature rise after 10 minutes RF heating tends to support the simulations presented here. However further work is clearly required to confirm MR safety before in vivo use.

\section{REFERENCES}

[1] H.L. Kantor, R.W. Briggs, R.S. Balaban, "In vivo ${ }^{31} \mathrm{P}$ nuclear magnetic resonance measurements in canine heart using a catheter-coil" Circ. Res. Vol. 55, pp. 261-266, Aug. 1984.

[2] K. Kandarpa, P. Jakab, S. Patz, F.J. Schoen, F.A. Jolesz, "Prototype miniature endoluminal MR imaging catheter" J. Vasc. Interv. Radiol. Vol. 4, pp. 419-427, May-June 1993.

[3] O. Ocali, E. Atalar, "Intravascular magnetic resonance imaging using a loopless catheter antenna" Magn. Reson. Med. Vol. 37, pp. 112-118, Jan. 1997.

[4] P.A. Bottomley, E. Atalar, R.F. Lee, K.A. Shunk, A. Lardo, "Cardiovascular MRI probes for the outside in and for the inside out" Magn. Reson. Mats. Phys. Biol. Med. Vol. 11, pp. 49-51, Nov. 2000.

[5] D. Crottet, R. Meuli, S. Wicky, J.J. van der Kink, "Reciprocity and sensitivity of opposed-solenoid endovascular MRI probes" J. Magn. Reson. Vol. 159, pp. 219-225, Dec. 2002.

[6] C.T. Farrar, V.J. Wedeen, J.L. Ackerman, "Cylindrical meanderline radiofrequency coil for intravascular magnetic resonance studies of atherosclerotic plaque" Magn. Reson. Med. Vol. 53, pp. 226-230, Jan. 2005.

[7] M. Burl, G.A. Coutts, D. Herlihy, R. Hill-Cottingham, J.E. Eastham, J.V. Hajnal, I.R. Young, "Twisted-pair RF coil suitable for locating the track of a catheter" Magn. Reson. Med. Vol. 41, pp. 636-638, Mar. 1999.

[8] Q. Zhang, M. Wendt, A.J. Aschoff, J.S. Lewin, J.L. Duerk, "A multielement RF coil for MRI guidance of interventional devices" J. Magn. Reson. Imag. Vol. 14, pp. 56-62, July 2001.

[9] S. Zuehlsdorff, R. Umathum, S. Volz, P. Hallscheidt, C. Fink, W. Semmler, M. Bock, "MR coil design for simultaneous tip tracking and curvature delineation of a catheter" Magn. Reson. Med. Vol. 52, pp. 214-218, July 2004.

[10] J.S. Hyde, R.J. Rilling, A. Jesmanowicz, "Passive decoupling of surface coils by pole insertion" J. Magn. Reson. Vol. 89, pp. 485-495, Oct. 1990

[11] W.A. Edelstein, C.J. Hardy, O.M. Mueller, "Electronic decoupling of surface coil receivers for NMR imaging and spectroscopy" J. Magn. Reson Vol. 67, pp. 156-161, Mar. 1986.

[12] G. Goubau, "On the excitation of surface waves" Proc. I.R.E. Vol. 40, pp. 865-868, July 1952 .

[13] M.K. Konings, L.W. Bartels, H.F.M. Smits, C.J.G. Bakker, "Heating around intravascular guidewires by resonating RF waves" J. Magn. Reson. Imag. Vol. 12, pp. 79-95, July 2000.

[14] C.-Y. Liu, K. Farahani, D.S.K. Lu, G. Duckwiler, A. Oppelt, "Safety of MRI-guided endovascular guidewire applications" J. Magn. Reson. Imag. Vol. 12, pp. 75-78, July 2000.

[15] W.R. Nitz, A. Oppelt, W. Renz, C. Manke, M. Lenhart, J. Link, "On the heating of linear conductive structures as guidewires and catheters in interventional MRI” J. Mag. Res. Imag. Vol. 13, pp. 105-114, Jan. 2001.

[16] S.M. Park, R. Kamondetdacha, A. Amjad, J.A. Nyenhuis, "MRI safety: RF-induced heating near straight wires" IEEE Trans. Magn. Vol. 41, pp. 4197-4199, Oct. 2005.

[17] A. Surowiec, S.S. Stuchly, L. Eidus, A. Swarup, "In-vitro dielectric properties of human tissue at radio frequencies" Phys. Med. Biol. Vol. 32, pp. 615-621, May. 1987.

[18] E. Atalar, "Safe coaxial cables" Proc. $7^{\text {th }}$ Ann. Meet. IRMRM, Philadelphia, PA, USA, pp. 1006, 24-28 May (1999)

[19] M.E. Ladd, H.H. Quick, "Reduction of resonant RF heating in intravascular catheters using coaxial chokes" Magn. Reson. Med. Vol. 43, pp. 615-619, Apr. 2000.

[20] S. Weiss, P. Vernickel, T. Schaeffter, V. Schulz, B. Gleich, "Transmission line for improved RF safety of interventional devices" Magn. Reson. Med. Vol. 54, pp. 182-189, July. 2005.

[21] A. Krafft, S. Müller, R. Umathum, W. Semmler, M. Bock, "B $_{1}$ fieldinsensitive transformers for RF-safe transmission lines" Magn. Reson. Mater. Phys. Vol. 19, pp. 257-266, Nov. 2006. 
[22] P.A. Bottomley, A. Kumar, W.A. Edelstein, J.P. Allen, P.V. Karmarkar, "Designing passive MRI-safe implantable conducting leads with electrodes" Med. Phys. Vol. 37, pp. 3828-3843, July 2010.

[23] R. Kozarek, "Biliary ERCP" Endoscopy Vol. 39, pp. 11-16, Jan. 2007.

[24] R.R.A. Syms, I.R. Young, M.M. Ahmad, S.D. Taylor-Robinson, M. Rea, "Magneto-inductive catheter receiver for magnetic resonance imaging" IEEE Trans. Biomed. Engng., Vol. 60, pp. 2421-2431, Sept. 2013.

[25] E. Shamonina, V.A. Kalinin, K.H. Ringhofer, L. Solymar "Magnetoinductive waveguide" Elect. Lett. Vol. 38, pp. 371-373, April 2002.

[26] R.R.A. Syms, I.R. Young, L. Solymar, T. Floume, "Thin-film magnetoinductive cables" J. Phys. D. Appl. Phys. Vol. 43, Art. 055102, Jan. 2010.

[27] R.R.A. Syms, T. Floume, "Electric coupling in strongly coupled magneto-inductive cable" $7^{\text {th }}$ Int. Cong. on Advanced Electromagnetic Metamaterials in Microwaves and Optics, Bordeaux, France, 16-21 Sept., 2013.

[28] A.E.H. Love, "Some electrostatic distributions in two dimensions" Proc. London Math. Soc. Vol. s2-22, 337-369, Jan. 1924.

[29] A. Bansal, B.P. Paul, K. Roy, "An analytic fringe capacitance model for interconnects using conformal mapping” IEEE Trans. Computer-aided Des. Integ. Circ. Syst. Vol. 25, pp. 2765-2774, Dec. 2006.

Khoonsake Seghoonthod is a $\mathrm{PhD}$ student in the EEE Dept., Imperial College London, UK, with a particular interest in the development of internal probes for magnetic resonance imaging of cholangiocarcinoma.

Richard Syms (SM 2002) is Professor of Microsystems Technology, EEE Dept., Imperial College London, UK, where he heads the Optical and Semiconductor Devices Group. He has published over 200 journal and conference papers on electromagnetic theory, sensors, metamaterials and medical imaging. He is a Fellow of the Royal Academy of Engineering, the Institute of Electrical Engineers and the Institute of Physics.

Ian Young is Senior Research Fellow, EEE Dept., Imperial College London, UK. He has published over 300 papers on MRI. His current interests are in-vivo MRI and MR-guided surgical robots. He is a Fellow of the Royal Society and a Fellow of the Royal Academy of Engineering, and winner of the Gold Medal of SMRM and the Sir Frank Whittle Prize. 